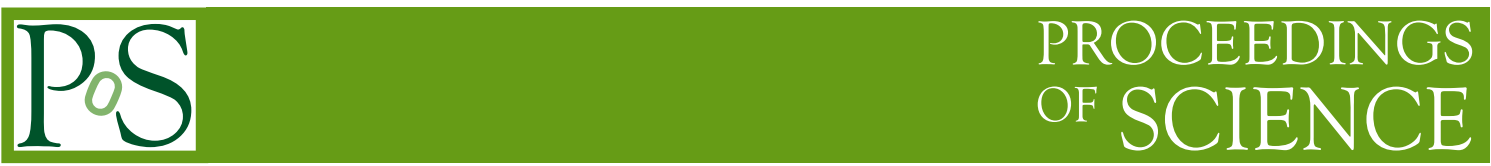

\title{
Operation and Performance of the AMS-02 Silicon Tracker
}

\author{
Saouter, P.*i \\ Université de Genève, 24 Quai Ernest-Ansermet, Genève, Switzerland. \\ E-mail: Pierre.Erwan. Saouterecern.ch
}

The AMS-02 magnetic spectrometer was installed on the International Space Station (ISS) in May 2011 and has since then been successfully collecting data. One of its main sub-detectors, the Silicon Tracker, determines the trajectory and absolute charge $|Z|$ of cosmic rays by multiple measurements of energy loss in nine layers of double sided silicon micro-strip detectors. In this contribution, we discuss the main challenges met by the operation of the Tracker in space. The resulting performances in terms of tracking efficiency and rigidity measurement are shown. We then describe the procedure that has been used to accurately calibrate the Tracker response to different nuclei species and optimize its performances in terms of charge resolution. The result is a resolution better than 0.3 charge units up to silicon and a mis-identification probability for carbon lower then $10^{-4}$ at $99 \%$ selection efficiency. The excellent charge identification performance of the Tracker is an important tool to study nuclear fragmentation in the detector. The precise estimation of this background of interacting events is particularly important for the precise measurements of cosmic ray nuclei fluxes and secondary to primary nuclei ratios that can help to better understand the production, acceleration and propagation of cosmic rays in the galaxy.

The 23rd International Workshop on Vertex Detectors,

15-19 September 2014

Macha Lake, The Czech Republic

\footnotetext{
* Speaker.

${ }^{\dagger}$ On behalf of the AMS-02 Tracker Collaboration.
} 


\section{The Alpha Magnetic Spectrometer AMS-02}

The Alpha Magnetic Spectrometer (AMS-02) is a state-of-the art particle physics detector operating as an external module on the International Space Station since May 2011. The purpose of the experiment is to perform accurate, high-statistics, long-duration measurements of the energy spectra of charged cosmic rays from $0.5 \mathrm{GeV}$ to few TeV. These measurements will help addressing fundamental questions like the search for dark matter and antimatter. Moreover, the absolute fluxes of most chemical species in cosmic rays, as well as their relative abundance, will help to improve models of acceleration and propagation of cosmic rays in the galaxy.

As depicted in Figure 1, at the core of the AMS-02 instrument is a permanent magnet of hollow cylindrical shape that provides a transverse field of $0.14 \mathrm{~T}$. A Silicon Tracker arranged in 9 planes placed at different vertical positions allows to reconstruct the particle trajectory and estimate the particle magnetic rigidity $R=p c / Z e$. The Tracker measures with an excellent resolution the magnitude of the charge of the traversing particle, offering up to nine independent measurements of the specific energy loss $d E / d x$. A Time of Flight system consisting of two plastic scintillator planes placed above and below the Inner Tracker provides the main trigger for the experiment. The ToF also determines if a particle is crossing the magnet in upward or downward direction and provides a sensitive measurement of the particle velocity with a $1 \%$ resolution, as well as good particle identification [2]. An array of anti-coincidence counters (ACC) surrounds the magnet inner bore and acts as a veto against particles traversing the detector sideways. At the top of the instrument, a Transition Radiation Detector (TRD) is used for its capability to distinguish between leptons and hadrons [3]. Below the lower ToF plane, a Ring Image Cherenkov detector (RICH) provides a high precision measurement of the particle velocity with a resolution at the per mille level, as well as a good particle identification power [4]. At the bottom of the instrument stands an Electromagnetic Calorimeter (ECAL) of 17 radiation length, serving accurate energy measurements for leptons and photons and a powerful $e^{+} / p$ rejection [5]. All subsystems have capabilities in measuring the absolute electric charge $Z$ [6]. More details on the various sub-detectors can be found in [7] and references therein.

\section{The Silicon Tracker}

The Tracker system is composed of 2284 double-sided silicon micro-strip sensors, with surface dimensions of $\sim 72 \times 41 \mathrm{~mm}^{2}$ and a $300 \mu \mathrm{m}$ thick n-doped silicon substrate. The sensors are assembled in basic functional elements called ladders. Each ladder is composed of 9 to 15 sensors, for a total of 192 ladders, and an active area of $6.75 \mathrm{~m}^{2}$. Each face of a sensor is implanted with strips running in orthogonal directions, providing a bi-dimensional measurement of the particle's position. The junction side (or p-side) is composed of $14 \mu \mathrm{m}$ wide $\mathrm{p}^{+}$doped strips, with an implantation pitch of $27.5 \mu \mathrm{m}$; the opposite ohmic side (the $\mathrm{n}$-side) has $40 \mu \mathrm{m}$ wide strips, with a larger implantation pitch of $104 \mu \mathrm{m}$. The strip metallization aluminium layer is slightly narrower than the implanted strips width, $12 \mu \mathrm{m}$ wide on the p-side and $36 \mu \mathrm{m}$ on the $\mathrm{n}$-side. The $\mathrm{n}$-side strip insulation is ensured by inserting a $\mathrm{p}^{+}$strip (p-stops) between each $\mathrm{n}^{+}$strip. The sensors are biased 


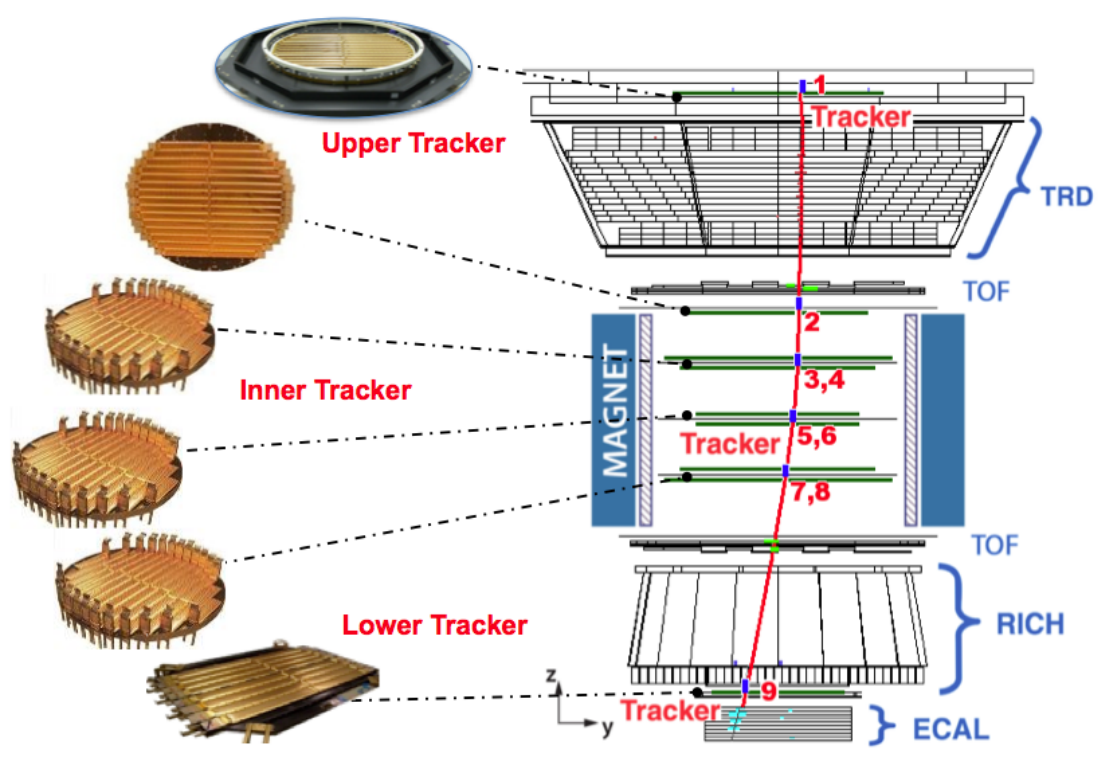

Figure 1: The figure displays the main sub-detectors composing AMS-02, presented in the bending $(y-z)$ plane of the magnetic field. Pictures of the 6 Tracker planes are also shown. The red line corresponds to a measured low energy proton. Picture taken from [1].

via the punch-through technique, the bias voltage being common to all sensors of a given ladder $[8,9]$. The $p$-sides of sensors are arranged such that the strips run in the longitudinal direction of the ladder and measure the bending coordinate $y$, while the n-side strips are orthogonal to the ladder length and measure the non-bending coordinate $x$.

The $\mathrm{p}$ and $\mathrm{n}$ side strips are separately connected in different daisy chains to the front-end electronics (TFE) which provides the coupling with the digitization system. On the p-side, the strips from different sensors of a given ladder are daisy-chained with micro-wire bonds. The final routing is achieved through a short $50 \mu \mathrm{m}$ thick Upilex cable directly glued on the silicon surface and connecting the strips of the ladder's first sensor to the p-side readout electronics. On the nside, since the strips are transverse to the ladder length, a long Upilex cable is glued to the ladder to redirect the signals from each sensor. The strips used to transmit the signals are composed of a gold layer covered by a copper layer. The 192 readout strips of sensors $1,3,5,7, \ldots$ are daisy chained through the Upilex cable and routed to a given groups of VA channels at the TFE, while the 192 strips of sensors $2,4,6,8, \ldots$ are routed to another group of VA channels.

Two separate electronic boards are used to readout the ladder, one for each side. The structure of the boards is the same on each side where the first stage is composed of decoupling capacitor chips (RCAMS) and the second stage is composed of pre-amplifier/shaper chips, the VA64_hdr. The RCAMS consist of 64 capacitors with a typical capacitance of $725 \mathrm{pF}$ whereas the interstrip capacitance is $2 \mathrm{pFcm}^{-1}$ and $1 \mathrm{pFcm}^{-1}$ for the p-side and n-side respectively. The VA64_hdr design is based on a high dynamic range, 64 channel chip, developed on the basis of the VA and Viking chips [11]. A total of 10(6) VA chips are used to read the signals from the p(n) side. Each VA readout channel consists of a charge sensitive amplifier, a CR-RC semi-Gaussian shaper and a sample-and-hold stage. The dynamic range for a single channel is around 100 times the signal of a 

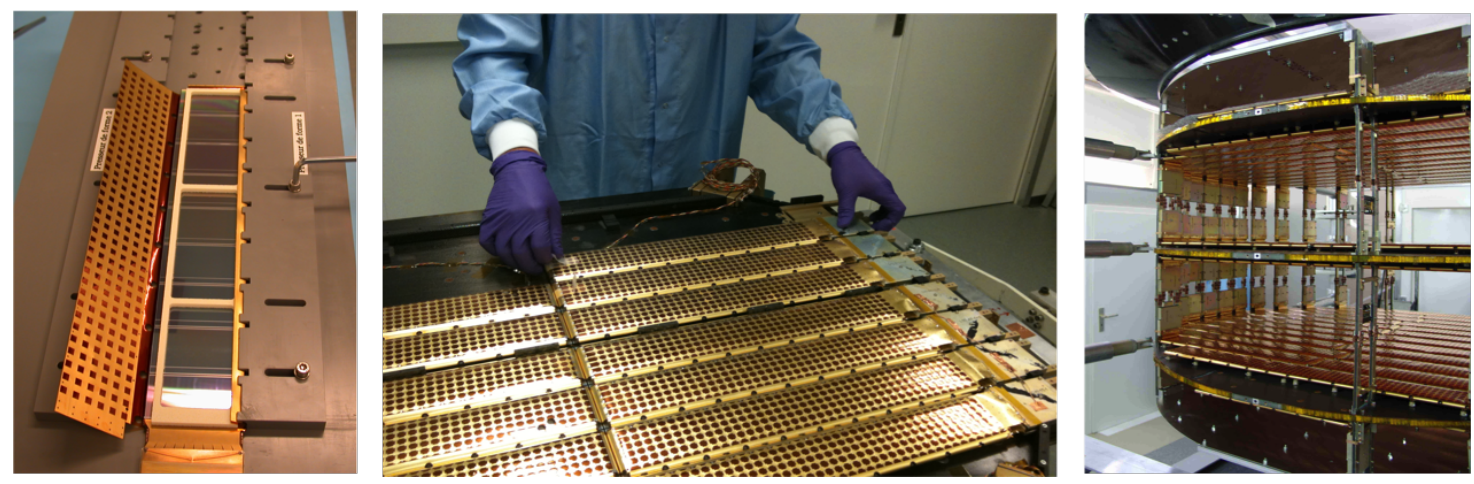

Figure 2: Left : picture of silicon ladder module, consisting in an assembly of sensors. Middle : picture of an external layer assembly. Right : the inner Tracker planes placed within the magnet bore.

minimum ionizing particle (MIP), with a linear response up to 75 MIPs. The power consumption criteria for each channel is $\sim 0.5 \mathrm{~mW}$.

The readout pitch is $110 \mu \mathrm{m}$ on the p-side and $208 \mu \mathrm{m}$ on the n-side which implies that only 1 out of 4 ( 1 out of 2 ) implantation strips are readout from the p-side (n-side). The difference in the number of implantation and readout strips is justified by the size and power consumption requirements for the operation of the payload in space. Considering capacitive coupling between the strips and charge sharing effects, the configuration discussed here does not necessarily imply a significant loss of resolution. The intrinsic position resolution is in fact $10 \mu \mathrm{m}$ in the bending direction (p-side) and $30 \mu \mathrm{m}$ in the non-bending one (n-side).

The main electronics for the signal digitization and online data reduction are grouped on the Tracker Data Reduction board (TDR). The analog signals are multiplexed in the TFE, amplified and sent through coaxial cables to three 12 bits Analog to Digital Converters (ADC). The digitization frequency is $5 \mathrm{MHz}$, a compromise between signal quality and the total readout time of about $80 \mu \mathrm{s}$. The data flow from the ADCs to the memory buffers is controlled by a Field Programmable Gate Array (FPGA) while a Digital Signal Processor (DSP) is programmed to collect the events from the buffers and apply either on-line calibration or data reduction procedures.

The ladders are arranged in 9 layers grouped in 6 planes, as shown in Figure 1. The 6 circular support planes are composed of a low density aluminum honeycomb enclosed in a thin carbon fiber structure. The total average thickness of a Tracker plane is about $1.8 \%$ of radiation length, making the Tracker a very transparent detector, thus minimizing the effects of multiple scattering. In Figure 2 are shown pictures of a silicon ladder (left), an external layer assembly (middle) and the Inner Tracker planes placed inside the magnet bore. Details about the Tracker construction and testing on ground can be found in $[8,12,13]$.

\section{Operation and Performance of the Tracker}

The operation of the Silicon Tracker in space is particularly challenging due to the high temperature variations that can be experienced within different time scales. The temperature in space 
varies as a function of the incident angle of sunlight, which depends on the solar beta angle ${ }^{1}$, the day and night passages of the ISS (1 orbit is accomplished every 93 minutes), the orientation of the ISS and the position of the ISS radiators, which can depend on special activities like attitude reconfigurations for visiting vehicles or maneuvers to re-boost the station or to avoid floating debris. Figure 3 shows the temperature variation with time of the TFE for different Tracker layers. Due to a dedicated Tracker Thermal Control System (TTCS), based on a mechanically pumped two phase $\mathrm{CO}_{2}$ cooling loop system, the Inner Tracker (red) is kept within $1{ }^{\circ} \mathrm{C}$ of its nominal operational temperature ${ }^{2}$. Since the layer 1 (green) and layer 9 (blue) only benefit a passive control of the temperature, the excursions are more significant, fluctuating within $10^{\circ} \mathrm{C}$.

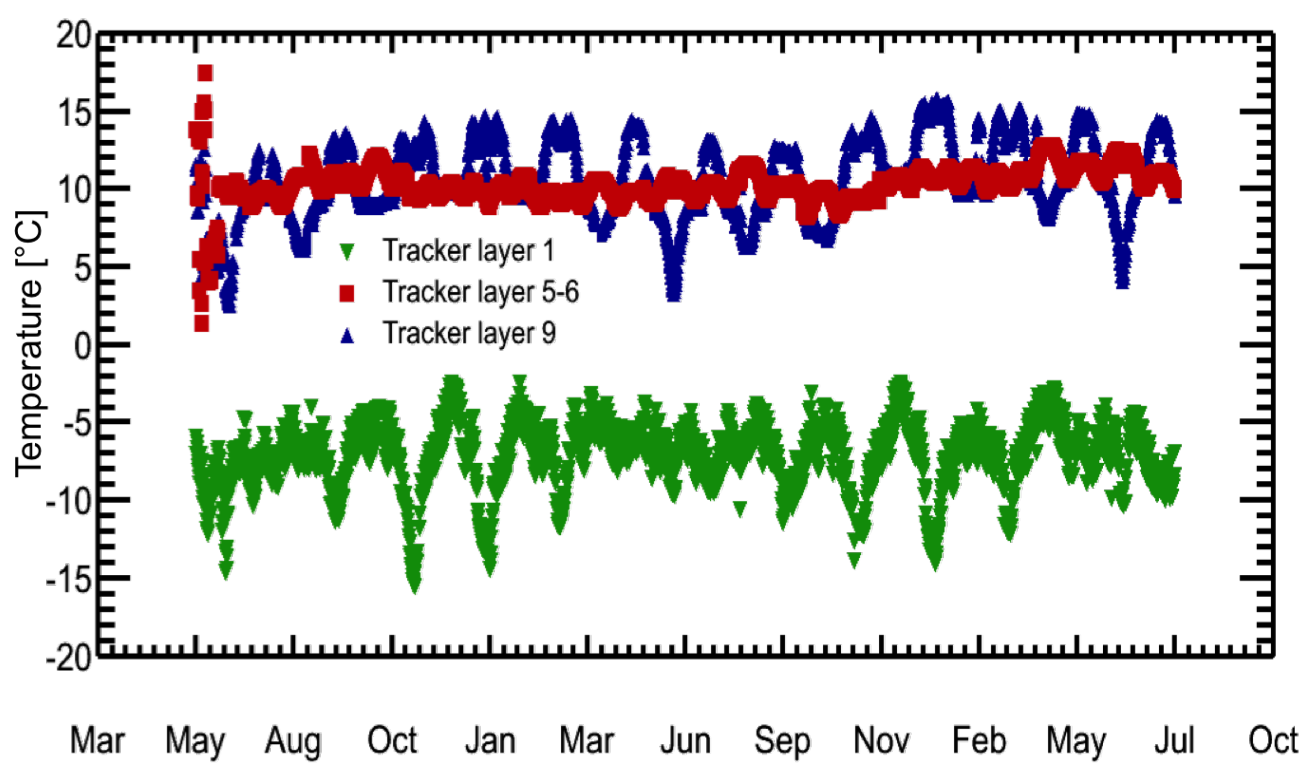

Figure 3: Temperature variation with time during the years 2011 and 2012, measured by different sensors thermally connected to the TFEs of the Inner, Upper and Lower Tracker. Plot from [14].

To take into account the high temperature excursions along different orbits, the Tracker is calibrated twice per orbit, every 46 minutes, when crossing the equator. On equatorial latitudes, the particle rate is minimum and thus the effect of events crossing the detector during the calibration are minimized. The procedure consists in the calculation of the pedestal and noise values for all the 200k readout channels of the Tracker. Figure 4 shows the average pedestal (top left) and noise (bottom left) values for both sides of all silicon ladders. Both values are stable with time, albeit small fluctuations in the first months of activation due to the commissioning of the detector in space. The increase of the average noise level on the $\mathrm{x}$-side, starting after January 2012, is due to changes in the calibration configuration and in the electronics. More details on the calibration procedure can be found in Refs $[9,14]$. The time stability and in-flight performances of the Silicon

\footnotetext{
${ }^{1}$ The angle between the ISS orbit plane and the sun vector and has the strongest impact on the temperature.

${ }^{2}$ The strong fluctuations of the Inner Tracker temperature in the first month of operations are due to the commissioning of the TTCS system.
} 
Tracker are discussed in Ref. [14].

The results of the calibration are used to trigger the acquisition of so-called clusters, a set of contiguous read-out strips with a signal above a given threshold. The threshold value is optimized in terms of particle detection efficiency and to minimize the number of clusters due to simple noise, such that the constraints on the total event size from AMS-02 imposed by the limited transmission bandwidth of the ISS are respected. The clusters set the basis for the particle track reconstruction in the detector [16]. The impinging coordinate of the particle is estimated by the center of gravity of the signals of the two higher signal strips ${ }^{3}$. The resolution is estimated to be $10(30) \mu \mathrm{m}$ on the $\mathrm{y}(\mathrm{x})$ coordinate for singly charged particles. The resolution improves for $Z>1$ particles, extending the range of detectable rigidities. Figure 4 shows the track reconstruction efficiency in function of rigidity (top) and time (bottom). The efficiency is measured using the Time of Flight instrument to select particles passing in the Tracker acceptance. The final efficiency is almost flat, both with rigidity and time, and above $92 \%$. The small drop in the efficiency seen after few months of operations (top right plot) is related to the increase in the average $\mathrm{x}$-side noise already discussed.
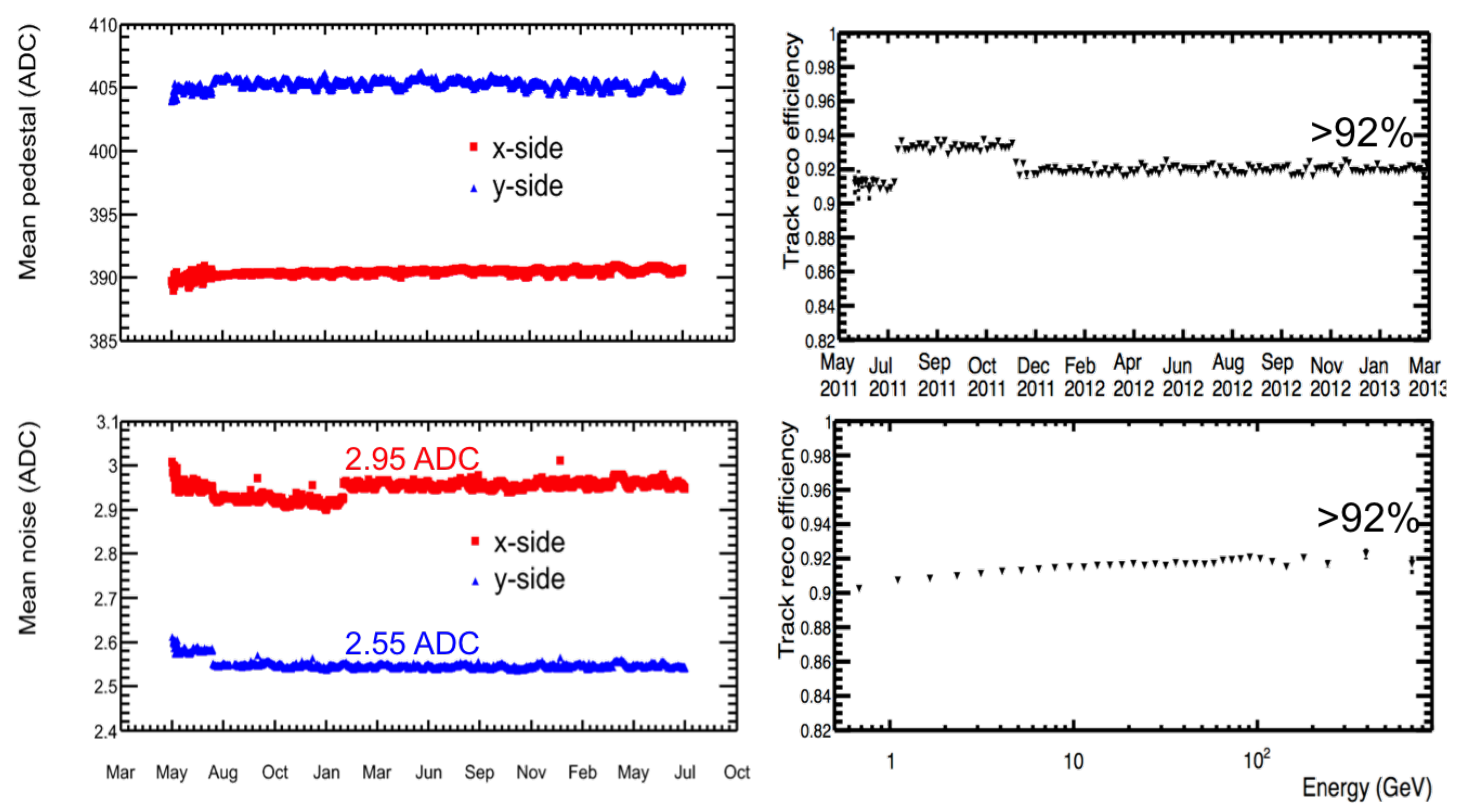

Figure 4: Left : Mean pedestal (top) and mean noise (bottom) values for the $\mathrm{x}$-side (ohmic n-side, red) and y-side (junction p-side, blue) of all silicon ladders during the years 2011 and 2012. Right : track reconstruction efficiency in function of time (top) during the years 2011 and 2012, and in function of rigidity (bottom). Plots taken from [14].

Due to the temperature variations on orbit, displacements of the outer Tracker layers of up to a few hundred microns were observed at a time scale of tens of minutes. A long-term movement of the sensors correlates with the evolution of the solar beta angle ( $\sim 2$ months cycle), while a shorter term variation is caused by the temperature cycles within a single orbit. In order to optimize the rigidity measurement in terms of resolution and maximum detectable rigidity (MDR), the relative

\footnotetext{
${ }^{3}$ For high inclinations of the incoming particle, using an additional strip in the calculation shows the best resolution.
} 
position of the sensors must be known with a precision better than $10 \mu \mathrm{m}$ at any moment. For that purpose, a static alignment of the Tracker sensors was performed both on ground and in space. In addition, a dynamic alignment of the outer layer sensors with respect to the Inner Tracker was performed in space. The alignment procedure is described in [15]. The final alignment error is estimated below $7 \mu \mathrm{m}$ which implies a maximum detectable rigidity (MDR) of $1.8 \mathrm{TV}$ for protons.

\section{Nuclei Identification}

The deposited ionization energy in a silicon sensor is proportional to the square of the particle charge $\left(d E / d x \propto Z^{2}\right)$, therefore allowing to distinguish different nuclei species. The analog readout and the high dynamic range of the front-end electronics of the Tracker ladders allow to identify nuclei from hydrogen up to iron and perhaps above ${ }^{4}$. The charge resolution of the Tracker is naturally degraded by a number of detector effects that need to be carefully taken into account and corrected for. In-flight calibration of the Tracker is done using the statistics accumulated over two years of operation, allowing to perform a precise equalization and linearization of the amplifier chips' responses. The details of the calibration procedure can be found in [18].

\subsection{Equalization and Linearization Procedure}

The total amplitude of a cluster (defined as the sum of the individual cluster strip signals) is first corrected for a cross-talk effect between neighboring readout channels. The amplitude is then normalized to an effective silicon thickness of $300 \mu \mathrm{m}$, using a multiplicative factor depending on the particle inclination. We then correct for differences in the response of the different VA chips. For each of the 3072 VA units, equalization factors are computed based on the signal response for the 3 most abundant nuclei species: hydrogen, helium and carbon.

One of the most subtle effect is related to the dependence of the total cluster amplitude on the impact position of the particle on the sensor and on its inclination, as can be appreciated in Figure 5 for a selected sample of helium nuclei measured by the x-side. The Impact Point (IP) is defined as the distance, in units of readout strip pitch, of the particle impact point on the sensor surface to the closest readout strip. For a particle impinging directly on a readout strip, $I P=0$, while $I P= \pm 0.5$ when the particle traverses in between two readout strips. The $\theta_{X Z}$ angle corresponds to the projection of the particle inclination on the $x-z$ plane of AMS- $02^{5}$.

The observed dependence can be understood in terms of a loss of collection efficiency when a particle traverses the sensor surface in between two readout strips, while the maximum charge collection efficiency appears when the particle impacts vertically on a readout strip. For the former case, at higher inclinations, the signal tends to be distributed to a larger number of strips which partially compensates the loss of collection and attenuates the effect. The total effect can amount to differences up to more than $30 \%$ for the lighter nuclei $(Z<6)$. As the charge increases, the

\footnotetext{
${ }^{4}$ Test beam results [13] have shown that the identification power extends above Iron. However, due to the very low flux of cosmic ray nuclei heavier than Iron, the final in-flight performance for these heavier species cannot not yet be confirmed.

${ }^{5}$ For studying the effect on the p-side, the relevant projection is on the $y-z$ plane.
} 
deposited energy is collected by an increasing number of readout strips and the effect tends to decrease. We produce 3-dimensional description plots of the type of Figure 5 for each nucleus and each sensor side which allow in turn to compute equalization factors for each bin of $I P$ and $\theta$.

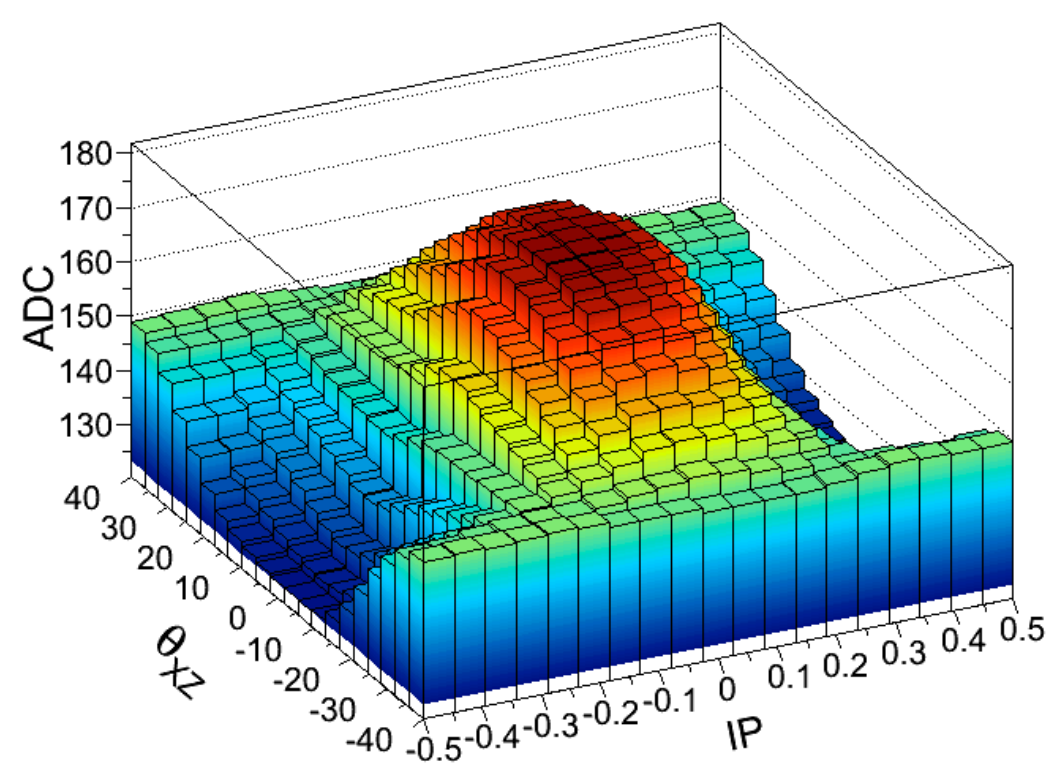

Figure 5: dependence of the raw signal on the impact position of the particle and its inclination for a selected sample of helium nuclei. The maximum of collection efficiency appears when the particle traverses at vertical incidence, impinging directly on a readout strip, i.e. $I P=0$ and $\theta_{X Z}\left(\theta_{Y Z}\right)=0$.

The dependence of the ionization energy loss upon the particle velocity is described by the Bethe-Bloch formula $d E / d x=Z^{2} f(\beta \gamma)$. In order to construct a single, energy independent, Tracker charge estimator, an effective parameterization has been derived. The universal $\beta \gamma$ parameter has been evaluated using both the ToF $\beta$ measurement at low energy and the Tracker rigidity measurement at higher energy. Effects of the slow-down of the particle due to energy losses in AMS-02 materials have also been included and the parameterization produced for all nuclei species up to iron $(Z=26)$.

Finally, a VA level linearity correction was implemented using the response from nuclei with $Z=1$ to $Z=26$.

\subsection{Charge Identification Performances}

Up to 18 estimations of the charge can be derived for a single particle traversing through all layers of the Tracker. These estimations can be combined into a unique estimator of the particle charge. The final Tracker charge estimator is shown in Figure 6. Nuclei up to Magnesium $(Z=16)$ can clearly be separated. Above, the Tracker separation power degrades due to saturation effects in the electronics. For the combined measurement, we reach a resolution (based on the width of a gaussian fits to individual nuclei peaks) at the level of 0.1 charge units (c.u.) for carbon and 
lower than 0.3 c.u. up to silicon. At $99 \%$ selection efficiency, the contamination from neighboring charges is estimated to be lower than $10^{-4}$ up to Oxygen $(\mathrm{Z}=8)$.

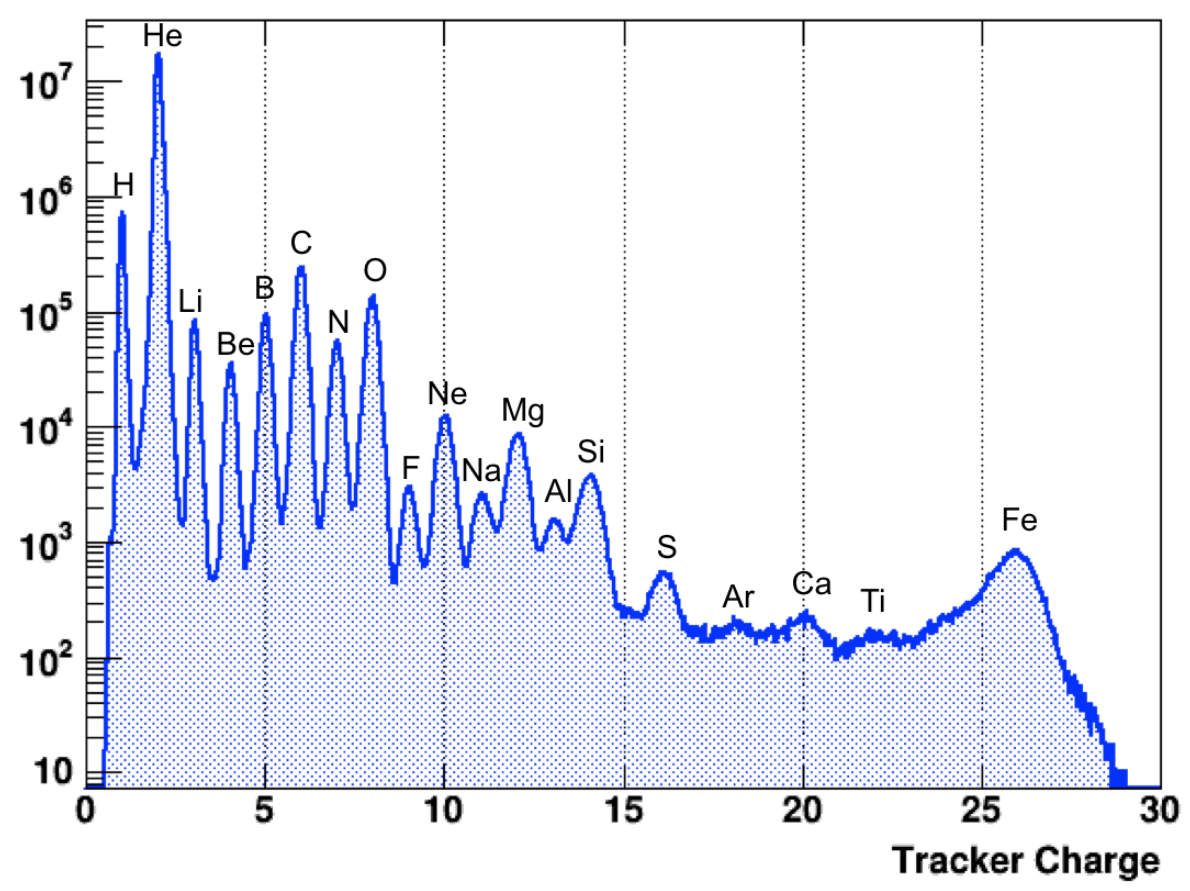

Figure 6: Final Tracker charge estimator obtained by combining up to 18 individual measurements of the particle charge. The contribution of hydrogen has been strongly suppressed in the data sample considered here.

\subsection{Chemical Composition Studies}

Figure 7 illustrates the importance of the good performance of the Tracker in terms of nuclei identification. The display of AMS-02 on the left shows a real event where a particle is identified in the Tracker layer 1 as oxygen $(Z=8)$ and as Carbon by the Inner Tracker, indicating that a nuclear fragmentation has occurred in the TRD or in the upper ToF plane. The black distribution in the middle plot shows the overall inner Tracker charge distribution accumulated over two years of statistics. The peaks from different nuclei species can clearly be distinguished. Based on a selection of pure carbon samples, indicated by the dashed blue band, the charge distribution of the layer 1 (upper blue distribution) allows to identify the contribution of heavier nuclei that have fragmented into carbon before reaching the Inner Tracker. Identifying such events is fundamental for the precise measurements of nuclei fluxes and secondary to primary nuclei ratios (like the Boron over Carbon ratio [19]) which are the most relevant to constrain models of acceleration and propagation of cosmic rays in the galaxy.

\section{Conclusion}

The AMS-02 detector has been operating as an external module on the International Space 


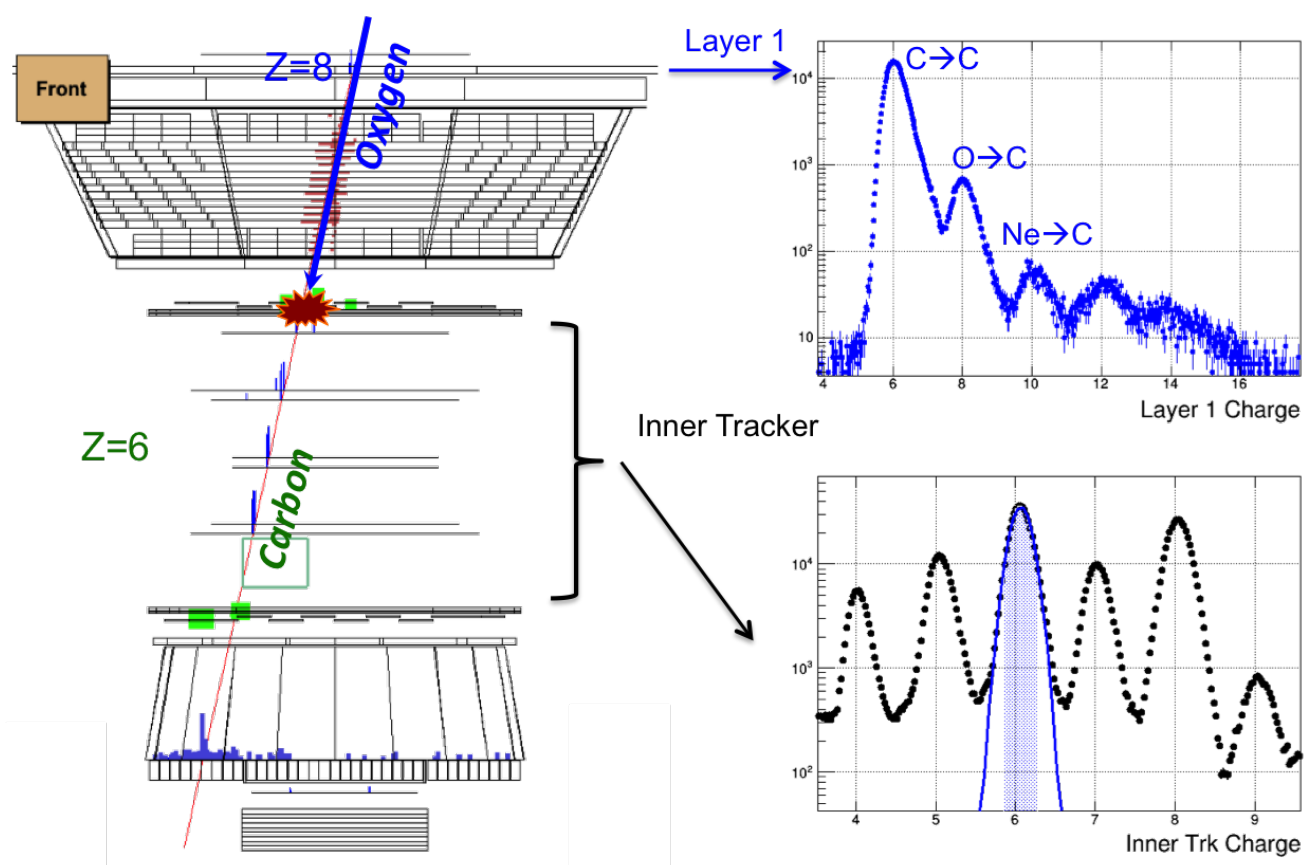

Figure 7: Nuclear fragmentation in the AMS-02 detector. See text for more details.

Station since more than three years. To account for the temperature variations on-orbit, dedicated calibration and alignment procedures have been developed. The overall performance of the Tracker in terms of tracking efficiency and rigidity measurement is high and stable with time. The procedure to optimize the performance of the Tracker in terms of nuclei identification has been discussed. After correcting for the most relevant effects degrading the charge measurement, we reach an excellent charge separation power up to magnesium $(Z=16)$. The mis-identification probability for carbon, at $99 \%$ selection efficiency, is lower than $10^{-4}$.

The excellent performance of the Tracker charge measurement is extremely useful to control fragmentation inside the detector. The estimation of this background is crucial for fundamental measurements such as secondary to primary nuclei ratios (for instance the boron over carbon ratio) used to better understand the production, acceleration and propagation of cosmic rays in the galaxy.

\section{References}

[1] A. Oliva, Proc. of Science, Vertex 2013 (2013).

[2] V. Bindi et al., NIM A, 743, 2014.

[3] H. Gast et al., Proc. of 33rd ICRC 1028 (2013).

[4] F. Giovacchini et al., Proc. of 33rd ICRC 1028 (2013).

[5] C. Adloff et al., NIM A, 714, 2013.

[6] N. Tomassetti et al., Proc. of 33rd ICRC 896 (2013).

[7] M. Aguilar et. al., Phys. Rev. Lett. 110, 141102 (2013), and references therein. 
[8] P. Azzarello, PhD Thesis, 2005.

[9] J. Alcaraz et al., NIM A, 593, 2008.

[10] M. Aguilar et al., Physical Review Letters, 110, 2013.

[11] E. Nygaard et al., NIM A, 506, 1991.

[12] W. J. Burger et al., Nucl. Instrum. Methods A 512 (2003) 517.

[13] J. Alpat et al., NIM A, 540, 1991.

[14] J. Bazo, et al., Proc. of 33rd ICRC 849 (2013).

[15] C. Delgado et al., Proc. of 33rd ICRC 849 (1260).

[16] P. Zuccon et al., Proc. of 33rd ICRC 1064 (2013).

[17] A. Oliva, PhD Thesis, 2006.

[18] P. Saouter, PhD Thesis, Université de Genève, 2014.

[19] A. Oliva et al., Proc. of 33rd ICRC 1266 (2013). 